\title{
Detachment of human endothelial cell sheets from thermo-responsive poly(NiPAAm-co-DEGMA) carriers
}

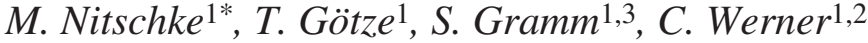 \\ ${ }^{1}$ Leibniz Institute of Polymer Research Dresden, Max Bergmann Center of Biomaterials, Hohe Str. 6, 01069 Dresden, \\ Germany \\ 2Institute of Biomaterials and Biomedical Engineering, University of Toronto, 5 King's College Road, Toronto, M5S 3G8 \\ Canada \\ ${ }_{3}^{3}$ present address: Oxyphen GmbH, Bautzner Landstrasse 45, 01454 Großerkmannsdorf, Germany
}

Received 31 May 2007; accepted in revised form 8 August 2007

\begin{abstract}
The technique of gently harvesting cells and cell sheets using stimuli-responsive cell culture carriers was applied to human umbilical vein cord endothelial cells (HUVEC). To meet the particular requirements of this demanding cell type, a copolymer consisting of $N$-isopropylacrylamide and diethyleneglycol methacrylate (poly(NiPAAm-co-DEGMA)) was combined with a fine-tuned protein pre-coating. Using this approach the detachment of HUVEC sheets was studied. Furthermore, the behavior of the extracellular matrix upon cell detachment was followed by protein staining. The results demonstrate the feasibility of harvesting HUVEC sheets from stimuli-responsive polymer layers and provide valuable options for the advanced engineering of vascular structures.
\end{abstract}

Keywords: smart polymers, polymer gels, poly( $N$-isopropylacrylamide), tissue engineering

\section{Introduction}

Polymers with a thermally stimulated volume phase transition like poly $(N$-isopropylacrylamide) (PNiPAAm) [1] and NiPAAm containing copolymers can be prepared on solid surfaces by various techniques [2-6]. The resulting coatings respond with swelling and collapsing to small changes in the environmental temperature and can be used as cell culture carriers for harvesting cells and cell sheets without enzymatic or mechanical treatments [7-10].

Vascular endothelial cells receive a lot of attention in tissue engineering strategies as they mediate the interaction of flowing blood with other tissues [11]. Control of endothelial cell adhesion and growth is therefore considered crucial for safety and performance of implants in the cardiovascular system such as vascular grafts or stents. However, endothelial- ization in vivo often fails and pre-seeding of implants in vitro is applied according to various schemes $[12,13]$. However, even the time-consuming procedure of growing endothelial cell linings directly on the implant is limited because of the lack of the characteristic functional structure of natural vasculature combining layers of smooth muscle cells and endothelial cells [14-17]. To this end, the preparation and subsequent alignment of cell sheets may provide interesting options: Pre-fabricated endothelial cell layers may be deposited on top of smooth muscle cell linings on - degradable or nondegadable - biomaterials and, by that, form a functional structure closely resembling vascular tissue. A defined procedure for the harvesting of endothelial cell sheets is an essential requirement for the implementation of this strategy. 
In the literature there are several reports on cultivation and detachment of endothelial cells from thermo-responsive carriers [18-21]. Copolymerization of NiPAAm with a hydrophobic comonomer (n-butyl methacrylate) [19] was shown to be an effective approach for adjusting the substrate properties. The main objective of the work presented here is another route to adapt the technique of thermo-responsive cell culture carriers to the particular requirements of human endothelial cells. Towards this goal, the physico-chemical properties were adjusted by combining a hydrophilic ethylene glycol containing comonomer with a fine-tuned pre-coating of fibronectin.

Cell culture carriers based on a statistical copolymer consisting of $\mathrm{N}$-isopropylacrylamide and diethyleneglycol methacrylate (poly(NiPAAm-coDEGMA)) [22] were prepared by plasma immobilization [23-26]. This technique allows to immobilize polymer films with a thickness of a few nanometers onto polymeric substrates like Teflon AF [27] by low pressure plasma treatment. At appropriate parameters covalent fixation is achieved while important properties like the thermoresponsive swelling and collapsing of polymer chains are preserved.

Ethylene glycol containing comonomers increase the phase transition temperature compared to the NiPAAm homopolymer [28]. Though this effect is rather small for poly(NiPAAm-co-DEGMA), the hydrophilic character of the diethylene glycol group improves cell detachment [10] and allows a more gentle handling of cell sheets upon harvest.

\section{Experimental}

\subsection{Materials}

Teflon AF1600 ${ }^{\mathrm{TM}}$ solution (6\% wt/wt) [27] was purchased from DuPont. Teflon AF is an amorphous copolymer based on 2,2 bistrifluoromethyl4,5-difluoro-1,3-dioxole and tetrafluoroethylene. FC75, a fluorocarbon solvent for Teflon AF, was obtained from $3 \mathrm{M}$.

Poly(NiPAAm-co-DEGMA) $\left(M_{n} 40000 \mathrm{~g} \cdot \mathrm{mol}^{-1}\right.$, $M_{w} 130000 \mathrm{~g} \cdot \mathrm{mol}^{-1}$, polydispersity $\sim 3$ by GPC), was synthesized by free radical polymerization. The ratio of the comonomers in the copolymer is 99.2:0.8 (NiPAAm:DEGMA) [22] (Figure 1).

For fibronectin (FN) immunostaining primary antibody anti FN rabbit (US Biological, Swampscott,

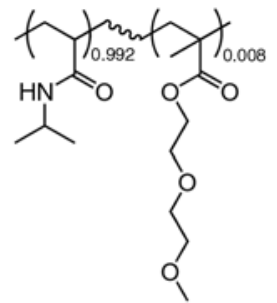

Figure 1. Structure of poly(NiPAAm-co-DEGMA)

Massachusetts) and secondary antibodies anti FN rabbit (Jackson Immuno Research Laboratories, West Grove, PA) were used.

\subsection{Teflon AF substrate preparation and hydrogel coating}

Thin films of Teflon AF were prepared by spin coating on microscopy cover slips $\left(24 \times 24 \mathrm{~mm}^{2}\right)$ and on silicon wafers $\left(15 \times 20 \mathrm{~mm}^{2}\right.$, oxide thickness $30 \mathrm{~nm}$, for ellipsometry). For that purpose, the Teflon AF solution as received was further diluted using FC75 to obtain a $1 \% \mathrm{wt} / \mathrm{wt}$ solution. After spin coating (maximum speed $3000 \mathrm{rpm}$, acceleration $3000 \mathrm{rpm} \cdot \mathrm{s}^{-1}, 30 \mathrm{~s}$ ), the Teflon AF films with a thickness of about $50 \mathrm{~nm}$ were annealed for $10 \mathrm{~min}$ utes at $110^{\circ} \mathrm{C}$. Subsequently, poly(NiPAAm-coDEGMA) was prepared on Teflon AF substrates by spin coating (maximum speed $5000 \mathrm{rpm}$, acceleration $5000 \mathrm{rpm} \cdot \mathrm{s}^{-1}, 30 \mathrm{~s}$ ) from a $0.25 \% \mathrm{wt} / \mathrm{wt}$ solution in chloroform $(99.8 \%$, Fluka). Teflon AF surfaces were pre-treated in argon plasma as described below for $120 \mathrm{~s}$ to obtain an appropriate wetting behavior.

\subsection{Plasma immobilization}

Poly(NiPAAm-co-DEGMA) thin films were immobilized using low pressure argon plasma. The plasma treatment was carried out in a computer controlled MicroSys apparatus by Roth \& Rau (Wüstenbrand, Germany). The cylindrical vacuum chamber, made of stainless steel, has a diameter of $350 \mathrm{~mm}$ and a height of $350 \mathrm{~mm}$. The base pressure obtained with a turbomolecular pump was $<10^{-7}$ mbar. On the top of the chamber a $2.46 \mathrm{GHz}$ electron cyclotron resonance (ECR) plasma source RR160 by Roth \& Rau with a diameter of $160 \mathrm{~mm}$ and a maximum power of $800 \mathrm{~W}$ was mounted. Argon (99.999\%, Messer Griesheim) was introduced into the active volume of the plasma source via a gas flow control system. When the plasma 
source was on, the pressure was measured by a capacitive vacuum gauge. The samples were introduced by a load-lock-system and placed on a grounded aluminum holder near the center of the chamber. The distance between the sample and the excitation volume of the plasma source was about $200 \mathrm{~mm}$. For the experiments of this work the following parameters were used: effective power $120 \mathrm{~W}$, argon gas flow $38 \mathrm{sccm}$, pressure $8 \cdot 10^{-3}$ mbar, treatment time $10 \mathrm{~s}$. After plasma treatment, the samples were rinsed in chloroform $(99.8 \%$, Fluka) for $1 \mathrm{~h}$ at room temperature and dried under vacuum.

To fabricate thermo-responsive cell culture carriers with lateral microstructures, the masking technique described in detail in [29] was used. Briefly, argon plasma treatment of a spin-coated poly(NiPAAmco-DEGMA) film was performed as described above with the sample covered by a transmission electron microscopy grid. The grid protected the copolymer layer underneath from plasma exposure which left this part soluble while the exposed part of the film was bonded to the Teflon AF surface. By rinsing with chloroform, the copolymer could be removed completely from the covered part leaving behind a microstructure.

\subsection{Spectrosopic ellipsometry}

Ellipsometric measurements were performed using a variable angle multiwavelength ellipsometer M-2000VI (J. A. Woollam Co., Inc.). It is a Diode Array Rotating Compensator Ellipsometer (DARCETM) in polarizer-compensator-sample-analyzer configuration equipped with an automatic computer-controlled goniometer and a horizontally mounted sample stage. The light source is a $50 \mathrm{~W}$ mercury lamp. For a given angle of incidence, the M-2000VI measures 500 wavelengths simultaneously covering the spectral range from 370$1700 \mathrm{~nm}$. Accurate measurements over the full $\Delta$ and $\Psi$ range were acquired $\left(\Delta=0^{\circ}-360^{\circ} ; \Psi=\right.$ $0^{\circ}-90^{\circ}$ ).

In case of dry samples, values from three angles of incidence, $65^{\circ}, 70^{\circ}$ and $75^{\circ}$ were acquired. To investigate swollen hydrogel layers, a solid-liquid cell (angle of incidence $68^{\circ}$ ) with de-ionized water (pH 6.5) was used. For the variation of temperature with a rate of $1 \mathrm{~K} \cdot \mathrm{min}^{-1}$, a computer controlled heating device for the solid-liquid cell was used.
To calculate the thickness and the optical properties of the polymer films under investigation, fit procedures based on optical multi-layer models were applied to the the ellipsometric data.

\subsection{Cell cultivation}

L929 mouse fibroblasts were obtained from DSMZ, Braunschweig, Germany and cultivated at $37^{\circ} \mathrm{C}, 5 \% \mathrm{CO}_{2}$ and $95 \%$ relative air moisture using RPMI medium (RPMI 1640; Pan Biotech GmbH, Aidenbach, Germany) containing 10\% fetal calf serum (FCS) and antibiotics.

Human endothelial cells from umbilical cord vein were collected according to the procedure suggested by Weis et al. [30]. Cells were grown at $37^{\circ} \mathrm{C}, 5 \% \mathrm{CO}_{2}$ and $95 \%$ relative air moisture on T25 culture dishes pre-coated with fibronectin (20 $\mu \mathrm{g} \cdot \mathrm{ml}^{-1}$ in phosphate buffered saline, PBS, $30 \mathrm{~min}$ at $37^{\circ} \mathrm{C}$ ) using endothelial cell growth medium (Promocell, Heidelberg, Germany) containing $2 \%$ FCS with supplement mix.

L929 mouse fibroblasts and HUVEC were harvested by trypsination and applied to the poly(NiPAAm-co-DEGMA) substrates. The substrates were allowed to swell for 24 hours at room temperature in PBS and subsequently conditioned at $37^{\circ} \mathrm{C}$. In case of HUVEC a pre-coating with fibronectin $\left(5 \mu \mathrm{g} \cdot \mathrm{ml}^{-1}\right.$ in PBS; $30 \mathrm{~min}$ at $\left.37^{\circ} \mathrm{C}\right)$ was applied. Subsequently, the solution was exchanged with the tempered cell culture medium. After a period of 30 minutes cells were seeded with a density of $1.5 \cdot 10^{5}$ cells per $\mathrm{cm}^{2}$ while keeping the temperature at $37^{\circ} \mathrm{C}$. The cultivation was proceeded until confluence was reached.

\subsection{Imaging techniques}

Microscopy images were obtained with an Axiovert 200 microscope by Zeiss. The microscope was equipped with a special incubator that allows cell cultivation $\left(37^{\circ} \mathrm{C}\right.$, air stream supplemented with $5 \% \mathrm{CO}_{2}$ ) and temperature variation while images are taken with a digital camera (AxioCam Colour). Axio Vision 3.1 software was used for image processing.

Fluorescence micrcroscopy images were obtained with an inverse fluorescence microscope Leica DMIRE2 equipped with a $40 \times$ oil immersion objective. Improvision 4.03 software was used for image processing. 
For immunostaining cells were fixed with $4 \%$ paraformaldehyde (Fluka) in PBS for $10 \mathrm{~min}$ and subsequently permeabilized with $0.5 \%$ Triton ${ }^{\circledR}$ X-100 (Fluka) in PBS for 10 min. Samples were stained with the primary and secondary antibodies (dilution 1:100 and 1:50 in PBS, retention time $45 \mathrm{~min}$ and $30 \mathrm{~min}$ respectively). After every step the samples were rinsed twice in PBS.

\section{Results and discussion}

\subsection{Characterization of the thermo- responsive cell culture carriers}

To study the multilayer polymer system, the optical properties of Teflon AF and poly(NiPAAm-coDEGMA) were determined. For that purpose thin films on silicon wafers were investigated by spectroscopic ellipsometry. Data sets were evaluated using a fit procedure based on an optical model consisting of the bulk silicon, the silicon oxide layer, and the respective polymer layer. Both polymers were found to be transparent, i. e., no absorption occurs in the wavelength range investigated $(k=0)$. The wavelength dependence of the refractive index was fitted using a two-parameter Cauchy equation (1):

$n(\lambda)=A_{n}+\frac{B_{n}}{\lambda^{2}}$

where $n$ : refractive index, $\lambda$ : wavelength, $A_{n}, B_{n}$ : Cauchy parameters [31]. The best fit of ellipsometric data provided $A_{n}=1.32$ and $B_{n} \leq 0.002$ for Teflon AF and $A_{n}=1.46$ and $B_{n} \leq 0.005$ for dry poly (NiPAAm-co-DEGMA) [10].

In the next step poly(NiPAAm-co-DEGMA) thin films covalently fixed by plasma immobilization on Teflon AF coated silicon substrates were immersed in distilled water at room temperature overnight and heated above the phase transition temperature. Subsequently, the temperature was decreased and increased repeatedly while the thickness and the effective refractive index of the swelling and collapsing hydrogel layer were monitored by spectroscopic ellipsometry. Above the phase transition temperature, constant values slightly higher than the dry film thickness were observed while an almost linear increase was found towards lower temperature values (Figure 2). The reversible switching has a amplitude of approximately $40 \mathrm{~nm}$ corresponding to a swelling ratio of about 3 with

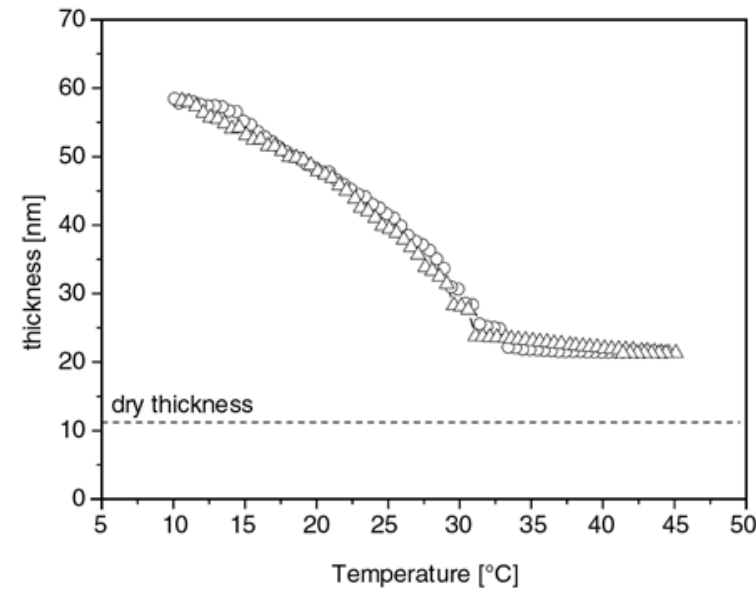

Figure 2. Reversible temperature dependent swelling behavior of poly(NiPAAm-co-DEGMA) thin films in water (circles cooling, triangles heating)

respect to the collapsed state of the poly(NiPAAmco-DEGMA) film. For the heating/cooling rate of $1 \mathrm{~K} \cdot \mathrm{min}^{-1}$ there is no significant hysteresis effect.

\subsection{Cell detachment experiments}

Experiments with L929 mouse fibroblasts, a simple and well-known cell line, were carried out on poly(NiPAAm-co-DEGMA) to ensure the comparability with previous studies on different thermoresponsive materials $[9,10]$. At $37^{\circ} \mathrm{C}$ cells adhere, spread, proliferate and reach confluence after $48 \mathrm{~h}$ (Figure 3 left) as observed on standard T25 cell culture dishes.

After temperature decrease to $23^{\circ} \mathrm{C}$ cells detach within a few minutes as a single sheet without enzymatic or mechanical treatment. In Figure 3 (right) the L929 layer retracts from the upper right to the lower left corner with no cells leaving behind on the thermo-responsive surface.

In the next step HUVECs were investigated. After seeding the cells and standard cultivation at $37^{\circ} \mathrm{C}$ for $48 \mathrm{~h}$ only single cells or small aggregates adhered to the copolymer surface. Cells did not show their characteristic morphology and did not form a confluent layer. To ensure initial cell adhesion and spreading, the collapsed thermo-responsive substrates were exposed to a fibronectin solution at $37^{\circ} \mathrm{C}$ for $30 \mathrm{~min}$. The concentration was varied from $3 \mu \mathrm{g} \cdot \mathrm{ml}^{-1}$ to $20 \mu \mathrm{g} \cdot \mathrm{ml}^{-1}$. An appropriate balance between initial cell adhesion and subsequent temperature driven cell detachment was found for a fibronectin concentration of $5 \mu \mathrm{g} \cdot \mathrm{ml}^{-1}$. 


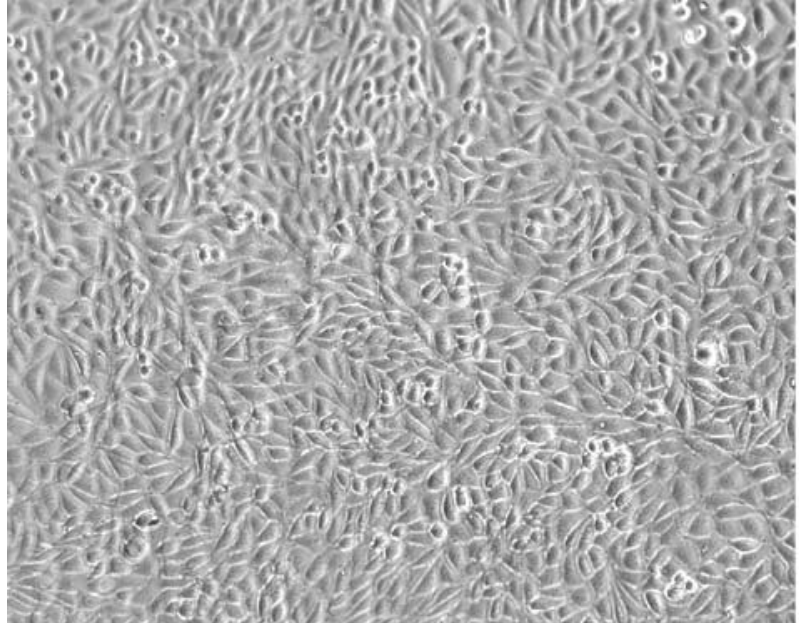

a)

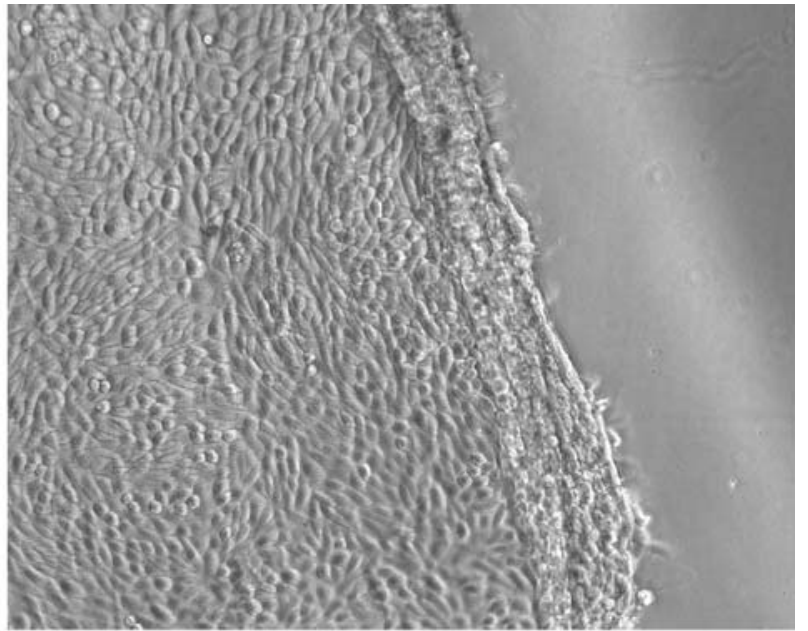

b)

Figure 3. L929 mouse fibroblast detachment from a poly(NiPAAm-co-DEGMA) substrate: Microscopy images $\left(873 \times 691 \mu \mathrm{m}^{2}\right)$ after standard cultivation at $37^{\circ} \mathrm{C}$ (left) and after temperature decrease (right)

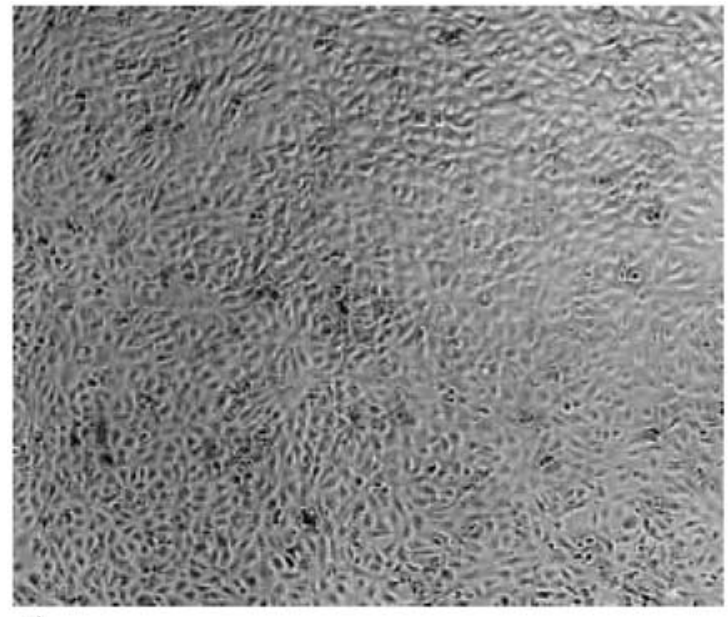

a)

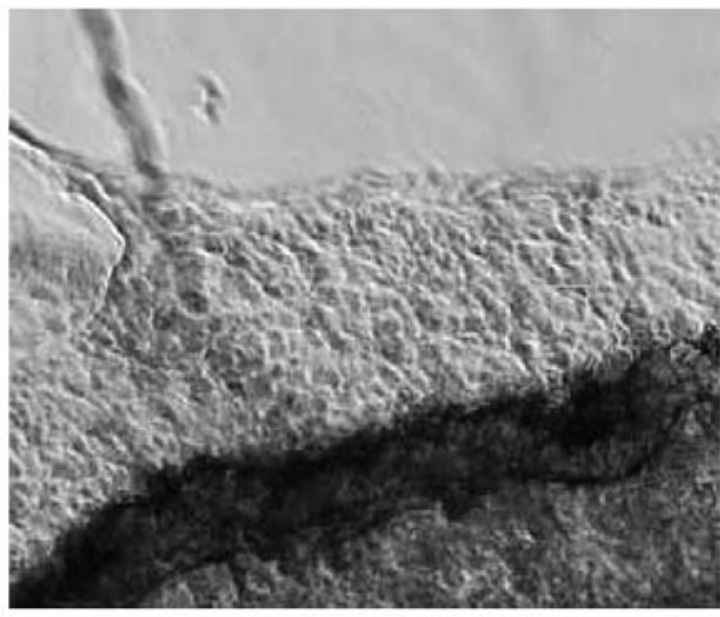

b)

Figure 4. HUVEC detachment from a poly(NiPAAm-co-DEGMA) substrate: Microscopy images $\left(512 \times 441 \mu \mathrm{m}^{2}\right)$ after standard cultivation at $37^{\circ} \mathrm{C}$ (left) and after temperature decrease (right)

Figure 4 shows a typical HUVEC detachment experiment on a pre-coated poly(NiPAAm-coDEGMA) substrate. For standard cultivation at $37^{\circ} \mathrm{C}$ on the $\mathrm{FN}$ exposed thermo-responsive carrier cells reach confluence after $20 \mathrm{~h}$ and show their characteristic cobblestone-like morphology (left). After temperature decrease to $23^{\circ} \mathrm{C}$ cells detach within a few hours as a single sheet (right). As in the case of L929 no cells are left behind on the swollen thermo-responsive substrate.

\subsection{Protein staining experiments}

Unraveling the fate of proteins of the extracellular matrix (ECM) on a thermo-responsive cell culture carrier during cell harvesting is a key to understand the mechanism of cell detachment [32]. Accord- ingly, the allocation of fibronectin as a major component of the ECM was investigated by immunofluorescence. For that purpose a substrate with lateral structures in the order of several $100 \mu \mathrm{m}$ (i. e. well above the dimension of a single cell) of poly(NiPAAm-co-DEGMA) was employed [29]. This allows a direct comparison of the cell behaviour on adhesive uncoated areas with thermoresponsive areas in the same image (Figure 5). A HUVEC layer grown at standard cultivation at $37^{\circ} \mathrm{C}$ (left) was cooled down below the phase transition temperature. Cells adhering on poly (NiPAAm-co-DEGMA) coated areas detached as described above (center). Subsequently, the lateral distribution of fibronectin was visualized by immunostaining (right). While in the adhesive areas cells and ECM are clearly visible, no fibronectin 

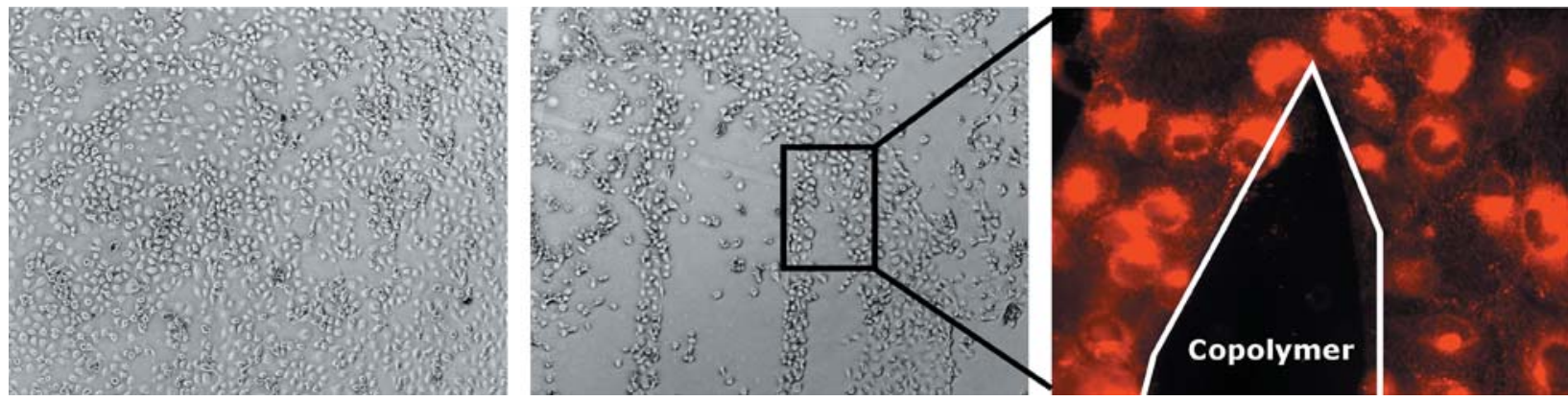

Figure 5. HUVEC detachment from a structured poly(NiPAAm-co-DEGMA) substrate: Microscopy images $\left(1747 \times 1385 \mu \mathrm{m}^{2}\right)$ after standard cultivation at $37^{\circ} \mathrm{C}$ (left) and after temperature decrease (center). Fluorescence microscopy image $\left(672 \times 512 \mu \mathrm{m}^{2}\right.$, fibronectin, Cy5) of the indicated region.

appears in areas that had been occupied by cells before. This agrees well with results from other studies [20, 21,33] showing that hardly any ECM remained on the surface of thermo-responsive cell culture carriers after cell detachment. This is an indication, that the ECM is removed together with endothelial cells providing coherence of the harvested cell sheets.

\section{Conclusions}

The technique of harvesting cells and cell sheets without enzymatic treatments using thermo-responsive cell culture carriers was applied to human endothelial cells. To meet the requirements of this cell type the physico-chemical properties of the thermo-responsive carriers were adjusted. Poly (NiPAAm-co-DEGMA), a polyethylene glycol containing hydrogel, was identified to provide an appropriate switching behaviour. The repeated swelling and collapsing of the material was characterized quantitatively by spectroscopic ellipsometry. A fine-tuned fibronectin pre-coating of the collapsed hydrogel surface ensures the initial adhesion and spreading of HUVEC. After reaching confluence, HUVEC sheets were detached successfully by decreasing the temperature to $23^{\circ} \mathrm{C}$. Immunostaining experiments prove the detachment of the extracellular matrix together with the cell sheet.

The reported results demonstrate the applicability of temperature-driven cell sheet harvesting to human endothelial cells grown in culture. As this cell type is of highest interest in the engineering of vascular structures future activities may utilize this approach for the design of advanced multilayered graft substitutes consisting of degradable biomaterials, smooth muscle cells and endothelial cells.

\section{Acknowledgements}

The authors thank R. Schulze and K. J. Eichhorn (Leibniz Institute of Polymer Research Dresden) for ellipsometry measurements and discussions.

\section{References}

[1] Schild H. G.: Poly (N-isopropylacrylamide)- experiment, theory and application. Progress in Polymer Science, 17, 163-249 (1992).

[2] Kwon O. H., Kikuchi A., Yamato M., Okano T.: Accelerated cell sheet recovery by co-grafting of PEG with PIPAAm onto porous cell culture membranes. Biomaterials, 24, 1223-1232 (2003).

[3] Cheng X. H., Canavan H. E., Stein M. J., Hull J. R., Kweskin S. J., Wagner M. S., Somorjai G. A., Castner D. G., Ratner B. D.: Surface chemical and mechanical properties of plasma-polymerized N-isopropylacrylamide. Langmuir, 21, 7833-7841 (2005).

[4] Curti P. S., de Moura M. R., Veiga W., Radovanovic E., Rubira A. F., Muniz E. C.: Characterization of PNIPAAm photografted on PET and PS surfaces. Applied Surface Science, 245, 223-233 (2005).

[5] Huang J., Wang X. L., Chen X. Z., Yu X. H.: Temperature-sensitive membranes prepared by the plasmainduced graft polymerization of $\mathrm{N}$-isopropylacrylamide into porous polyethylene membranes. Journal of Applied Polymer Science, 89, 3180-3187 (2003).

[6] Lin Z., Xu T. W., Zhang L.: Radiation-induced grafting of $\mathrm{N}$-isopropylacrylamide onto the brominated poly(2,6-dimethyl-1,4-phenylene oxide) membranes. Radiation Physics and Chemistry, 75, 532-540 (2006).

[7] Yamato M., Okano T.: Cell sheet engineering. Materials Today, 7, 42-47 (2004).

[8] Yang J., Yamato M., Kohno C., Nishimoto A., Sekine H., Fukai F., Okano T.: Cell sheet engineering: Recreating tissues without biodegradable scaffolds. Biomaterials, 26, 6415-6422 (2005).

[9] Schmaljohann D., Oswald J., Jorgensen B., Nitschke M., Beyerlein D., Werner C.: Thermo-responsive PNiPAAm-g-PEG films for controlled cell detachment. Biomacromolecules, 4, 1733-1739 (2003). 
[10] Nitschke M., Gramm S., Götze T., Valtink M., Drichel J., Voit B., Engelmann K., Werner C.: Thermoresponsive poly(NiPAAm-co-DEGMA) substrates for gentle harvest of human corneal endothelial cell sheets. Journal of Biomedical Materials Research A, 80, 1003-1010 (2007).

[11] Werner C., Maitz M., Sperling C.: Current strategies towards hemocompatible coatings. Journal of Materials Chemistry, 17, 3376-3384 (2007).

[12] Nerem R. M.: Tissue engineering of the vascular system. Vox Sanguinis, 87, 158-160 (2004).

[13] Gong Z. D., Niklason L. E.: Blood vessels engineered from human cells. Trends in Cardiovascular Medicine, 16, 153-156 (2006).

[14] Berglund J. D., Galis Z. S.: Designer blood vessels and therapeutic revascularization. British Journal of Pharmacology, 140, 627-636 (2003).

[15] Bhattacharya V., Cleanthis M., Stansby G.: Preventing vascular graft failure: Endothelial cell seeding and tissue engineering. Vascular Disease Prevention, 2, 21-27 (2005).

[16] Nerem R. M., Seliktar D.: Vascular tissue engineering. Annual Review of Biomedical Engineering, 3, 225243 (2001).

[17] Stegemann J. P., Hong H., Nerem R. M.: Mechanical, biochemical, and extracellular matrix effects on vascular smooth muscle cell phenotype. Journal of Applied Physiology, 98, 2321-2327 (2005).

[18] Kushida A., Yamato M., Konno C., Kikuchi A., Sakurai Y., Okano T.: Decrease in culture temperature releases monolayer endothelial cell sheets together with deposited fibronectin matrix from temperatureresponsive culture surfaces. Journal of Biomedical Materials Research, 45, 355-362 (1999).

[19] Tsuda Y., Kikuchi A., Yamato M., Sakurai Y., Umezu M., Okano T.: Control of cell adhesion and detachment using temperature and thermoresponsive copolymer grafted culture surfaces. Journal of Biomedical Materials Research, Part A, 69, 70-78 (2004).

[20] Canavan H. E., Cheng X. H., Graham D. J., Ratner B. D., Castner D. G.: Cell sheet detachment affects the extracellular matrix: A surface science study comparing thermal liftoff, enzymatic, and mechanical methods. Journal of Biomedical Materials Research, Part A, 75, 1-13 (2005).

[21] Canavan H. E., Cheng X. H., Graham D. J., Ratner B. D., Castner D. G.: Surface characterization of the extracellular matrix remaining after cell detachment from a thermoresponsive polymer. Langmuir, 21, 1949-1955 (2005).
[22] Gramm S., Komber H., Schmaljohann D.: Copolymerization kinetics of $\mathrm{N}$-isopropylacrylamide and diethylene glycol monomethylether monomethacrylate determined by online NMR spectroscopy. Journal of Polymer Science, Part A: Polymer Chemistry, 43, 142-148 (2005).

[23] Nitschke M., Zschoche S., Baier A., Simon F., Werner C.: Low pressure plasma immobilization of thin hydrogel films on polymer surfaces. Surface and Coatings Technology, 185, 120-125 (2004).

[24] Nitschke M., König U., Lappan U., Minko S., Simon F., Zschoche S., Werner C.: Low pressure plasmabased approaches to fluorocarbon polymer surface modification. Journal of Applied Polymer Science, 103, 100-109 (2007).

[25] Sheu M. S., Hoffman A. S., Feijen J.: A glow-discharge treatment to immobilize poly(ethylene oxide) poly(propylene oxide) surfactants for wettable and nonfouling biomaterials. Journal of Adhesion Science and Technology, 6, 995-1009 (1992).

[26] Terlingen J. G. A., Feijen J., Hoffman A. S.: Immobilization of surface-active compounds on polymer supports using a gas-discharge process. Journal of Biomaterials Science: Polymer Edition, 4, 31-33 (1992).

[27] Korinek P. M.: Amorphous fluoropolymers- a newgeneration of products. Macromolecular Symposia, 82, 61-65 (1994).

[28] Schmaljohann D., Beyerlein D., Nitschke M., Werner C.: Thermo-reversible swelling of thin hydrogel films immobilized by low-pressure plasma. Langmuir, 20, 10 107-10 114 (2004).

[29] Schmaljohann D., Nitschke M., Schulze R., Eing A., Werner C., Eichhorn K. J.: In situ study of the thermoresponsive behavior of micropatterned hydrogel films by imaging ellipsometry. Langmuir, 21, 2317 2322 (2005).

[30] Weis J. R., Sun B., Rodgers G. M.: Improved method of human umbilical arterial endothelial cell culture. Thrombosis Research, 61, 171-173 (1991).

[31] Fattorini H. O., Kerber A.: The Cauchy problem; Encyclopedia of mathematics and its applications. Cambridge University Press, Cambridge (1984).

[32] Cheng X., Canavan H. E., Graham D. J., Castner D. G., Ratner B. D.: Temperature dependent activity and structure of adsorbed proteins on plasma polymerized $\mathrm{N}$-isopropyl acrylamide. Biointerphases, 1, 61-72 (2006).

[33] Yamato M., Konno C., Kushida A., Hirose M., Utsumi M., Kikuchi A., Okano T.: Release of adsorbed fibronectin from temperature-responsive culture surfaces requires cellular activity. Biomaterials, 21, 981986 (2000). 